\title{
ZERO-CYCLES ON SELF-PRODUCTS OF VARIETIES: SOME ELEMENTARY EXAMPLES VERIFYING VOISIN'S CONJECTURE
}

\author{
ROBERT LATERVEER
}

\begin{abstract}
An old conjecture of Voisin describes how zero-cycles on a variety $X$ should behave when pulled-back to the self-product $X^{m}$ for $m$ larger than the geometric genus of $X$. Using complete intersections of quadrics, we give examples of varieties in any dimension and with arbitrarily high geometric genus that verify Voisin's conjecture.
\end{abstract}

\section{INTRODUCTION}

Given a smooth projective variety $X$ over $\mathbb{C}$, let $A^{i}(X)_{\mathbb{Z}}:=C H^{i}(X)_{\mathbb{Z}}$ denote the Chow groups of $X$ (i.e. the groups of codimension $i$ algebraic cycles on $X$ with $\mathbb{Z}$-coefficients, modulo rational equivalence [7]). Let $A_{\text {hom }}^{i}(X)_{\mathbb{Z}}$ denote the subgroup of homologically trivial cycles.

The Bloch-Beilinson-Murre conjectures form a kind of Rosetta Stone, allowing to translate cohomological statements into conjectural Chow-theoretic statements [8], [9], [21], [10], [22], [29]. The following particular instance of such a translation was first formulated by Voisin:

Conjecture 1.1 (Voisin 1993 [28]). Let $X$ be a smooth projective $n$-dimensional variety with $H^{j}\left(X, \mathcal{O}_{X}\right)=0$ for $0<j<n$. Let $m$ be an integer strictly larger than the geometric genus $p_{g}(X):=\operatorname{dim} H^{n}\left(X, \mathcal{O}_{X}\right)$. Then for any zero-cycles $a_{1}, \ldots, a_{m} \in A_{\text {hom }}^{n}(X)_{\mathbb{Z}}$, there is an equality

$$
\sum_{\sigma \in \mathfrak{S}_{m}}(\operatorname{sgn}(\sigma))^{n+1} a_{\sigma(1)} \times \cdots \times a_{\sigma(m)}=0 \text { in } A^{m n}\left(X^{m}\right)_{\mathbb{Z}} .
$$

(Here $\mathfrak{S}_{m}$ is the symmetric group on m elements, and $\operatorname{sgn}(\sigma)$ is the sign of the permutation $\sigma$. The notation $a_{1} \times \cdots \times a_{m}$ is shorthand for the zero-cycle $p_{1}^{*}\left(a_{1}\right) \cdot p_{2}^{*}\left(a_{2}\right) \cdots p_{m}^{*}\left(a_{m}\right)$ on $X^{m}$, where the $p_{j}: X^{m} \rightarrow X$ are the various projections.)

This conjecture is the translation in Chow-language of the fact that the Hodge structure

$$
\wedge^{m} H^{n}(X, \mathbb{Q}) \subset H^{m n}\left(X^{m}, \mathbb{Q}\right)
$$

has Hodge coniveau $>0$ for $m>p_{g}(X)$ (see [28] or [29, Section 4.3.5.2] for more detailed motivation).

In case $p_{g}(X)=0$, Conjecture 1.1 predicts that $A^{n}(X)_{\mathbb{Z}} \cong \mathbb{Z}$ (this is a form of Bloch's conjecture [3] ). In case $p_{g}(X)=1$ (e.g., $X$ is a Calabi-Yau variety), the conjecture takes on a particularly appealing form: it predicts that any 2 degree 0 zero-cycles $a, a^{\prime} \in A_{h o m}^{n}(X)_{\mathbb{Z}}$ verify

$$
a \times a^{\prime}=(-1)^{n} a^{\prime} \times a \text { in } A^{2 n}(X \times X)_{\mathbb{Z}} .
$$

2010 Mathematics Subject Classification. Primary 14C15, 14C25, 14 C30.

Key words and phrases. Algebraic cycles, Chow groups, motives, Voisin conjecture, complete intersections of quadrics. 
This is still open for a general K3 surface (in fact, I am not aware of a K3 surface with Picard number $<9$ which is known to verify Voisin's conjecture). Examples of surfaces of geometric genus 1 verifying the conjecture (or a variant conjecture) are given in [28], [12], [14], [15], [30]. Examples of other varieties verifying the conjecture are given in [28], [18], [13], [16], [17], [19], [2], [20], [27], [5].

The main result of this note is that Voisin's conjecture is true for certain complete intersections of quadrics:

Theorem 1.2. Given $g, r \in \mathbb{N}$ and distinct numbers $\lambda_{0}, \ldots, \lambda_{2 g+1} \in \mathbb{C}$, let $X \subset \mathbb{P}^{2 g+1}(\mathbb{C})$ be the complete intersection defined by

$$
\begin{cases}x_{0}^{2}+x_{1}^{2}+\cdots \cdots \cdots+x_{2 g+1}^{2} & =0 \\ \lambda_{0} x_{0}^{2}+\lambda_{1} x_{1}^{2}+\cdots+\lambda_{2 g+1} x_{2 g+1}^{2} & =0, \\ \lambda_{0}^{2} x_{0}^{2}+\lambda_{1}^{2} x_{1}^{2}+\cdots+\lambda_{2 g+1}^{2} x_{2 g+1}^{2} & =0, \\ \vdots & \\ \vdots & \\ \lambda_{0}^{r} x_{0}^{2}+\lambda_{1}^{r} x_{1}^{2}+\cdots+\lambda_{2 g+1}^{r} x_{2 g+1}^{2} & =0 .\end{cases}
$$

Then $X$ is a smooth projective variety, and Conjecture 1.1 is true for $X$.

These complete intersections are admittedly very special (and unfortunately the argument proving Theorem 1.2 does not apply to general complete intersections of quadrics), but at least they provide examples of any dimension, and with $p_{g}$ arbitrarily high, verifying Voisin's conjecture. The complete intersections of Theorem 1.2 are rather similar to the Calabi-Yau varieties of [20]: both are related to products of curves, and hence to abelian varieties. Thus, to prove Theorem 1.2 we can reduce to a problem concerning zero-cycles on abelian varieties; this last problem can be solved thanks to recent work of Vial [27].

As a consequence of Theorem 1.2, certain instances of the generalized Hodge conjecture are verified:

Corollary 1.3. Let $X$ be as in Theorem 1.2 and $m>p_{g}(X)$. The sub-Hodge structure

$$
\wedge^{m} H^{n}(X, \mathbb{Q}) \subset H^{m n}\left(X^{m}, \mathbb{Q}\right)
$$

is supported on a divisor.

Conventions. In this note, the word variety will refer to a reduced irreducible scheme of finite type over $\mathbb{C}$. A subvariety is a (possibly reducible) reduced subscheme which is equidimensional.

Unless indicated otherwise, all Chow groups will be with rational coefficients: we will denote by $A_{j}(X)$ (resp. $A_{j}(X)_{\mathbb{Z}}$ ) the Chow group of $j$-dimensional cycles on $X$ with $\mathbb{Q}$-coefficients (resp. $\mathbb{Z}$-coefficients); for $X$ smooth of dimension $n$ the notations $A_{j}(X)$ and $A^{n-j}(X)$ are used interchangeably. The notation $A_{h o m}^{j}(X)$ will be used to indicate the subgroups of homologically trivial cycles.

We will write $\mathcal{M}_{\text {rat }}$ (and $\mathcal{M}_{\text {hom }}$ ) for the contravariant category of pure motives with respect to rational equivalence (resp. homological equivalence), as in [24], [22]. 


\section{THE PROOF}

This section contains the proof of the main result:

Proof. (of Theorem 1.2.) This is based on results of Terasoma, who has made an in-depth study of this kind of complete intersection [25, Sections 2.4, 2.5 and 2.6] (NB: in loc. cit., the variety $X$ is denoted $X_{r, g}$ which is (by definition) the same as $X_{r, 2 g+2,2}$ ).

First, the non-singularity of $X$ is readily checked using the Vandermonde determinant (cf. [25, Proposition 2.4.1]).

Next, to verify Conjecture 1.1 for $X$, we need to understand the Chow group of zero-cycles $A^{n}(X)_{\mathbb{Z}}$ (where we write $n:=2 g-r$ for the dimension of $X$ ). Thanks to a theorem of Roitman [23], it suffices to prove that Conjecture 1.1 is true for $A^{n}(X)$, the Chow group with $\mathbb{Q}$ coefficients. From Terasoma's work [25, Corollary 2.5.3], we know there exist a finite number of hyperelliptic curves $C_{\chi}$ and a correspondence $\Gamma$ inducing an isomorphism in cohomology

$$
\Gamma_{*}: H_{\text {prim }}^{n}(X, \mathbb{Q}) \stackrel{\cong}{\rightarrow} \bigoplus_{\chi} \wedge^{n} H^{1}\left(C_{\chi}, \mathbb{Q}\right) \text {. }
$$

Because curves and complete intersections verify the Lefschetz standard conjecture, we know that the inverse is also induced by a correspondence. (This is well-known, cf. for instance [26, Proof of Proposition 1.1], where I first learned this. In a nutshell, the argument is as follows: the Lefschetz standard conjecture for $X$ implies that there is a correspondence $s \in A^{n}(X \times X)$ inducing a polarization on $H_{\text {prim }}^{n}(X, \mathbb{Q})$. A Hodge-theoretical argument [26, Lemma 1.6] then gives that $\alpha:=\Gamma \circ s \circ{ }^{t} \Gamma$ induces an automorphism of $\bigoplus_{\chi} \wedge \wedge^{n} H^{1}\left(C_{\chi}, \mathbb{Q}\right)$. By the CayleyHamilton theorem, the inverse to $\alpha_{*}$ is given by $P(\alpha)_{*}$ for some rational polynomial $P$. Then $\Gamma \circ s \circ{ }^{t} \Gamma \circ P(\alpha)$ acts as the identity on $\bigoplus_{\chi} \wedge \wedge^{n} H^{1}\left(C_{\chi}, \mathbb{Q}\right)$, and so the correspondence $s \circ^{t} \Gamma \circ P(\alpha)$ gives an inverse to $\Gamma_{*}$. )

This means that there is an isomorphism of homological motives

$$
[\Gamma]: h_{\text {prim }}^{n}(X) \stackrel{\cong}{\rightarrow} \bigoplus_{\chi} \operatorname{Sym}^{n} h^{1}\left(C_{\chi}\right) \text { in } \mathcal{M}_{\mathrm{hom}} .
$$

Let $h_{\text {prim }}^{n}(X) \in \mathcal{M}_{\text {rat }}$ be the Chow motive such that

$$
h(X)=h_{\text {prim }}^{n}(X) \oplus \bigoplus \mathbb{1}(*) \text { in } \mathcal{M}_{\text {rat }}
$$

(that is, $h_{\text {prim }}^{n}(X)$ is defined by the projector $\pi_{X}^{n, \text { prim }}:=\Delta_{X}-\frac{1}{2^{r+1}} \sum_{j} h^{j} \times h^{n-j}$, where $h \in$ $A^{1}(X)$ denotes a hyperplane section). The variety $X$ is isomorphic to a quotient $D^{2 g-r} / G$, where $D$ is a smooth curve [25, Theorem 2.4.2], and so $X$ is Kimura finite-dimensional [10]. We recall Kimura's fundamental nilpotence theorem [10], which implies that the functor from finitedimensional Chow motives to homological motives is conservative, i.e. detects isomorphisms. The isomorphism (2) can thus be upgraded to an isomorphism of Chow motives

$$
h_{\text {prim }}^{n}(X) \stackrel{\cong}{\rightarrow} \bigoplus_{\chi} \operatorname{Sym}^{n} h^{1}\left(C_{\chi}\right) \text { in } \mathcal{M}_{\text {rat }} .
$$

Let $J_{\chi}:=\operatorname{Jac}\left(C_{\chi}\right)$ denote the Jacobian of the curve $C_{\chi}$. There are isomorphisms $h^{1}\left(C_{\chi}\right) \cong$ $h^{1}\left(J_{\chi}\right)$. Moreover, $\operatorname{Sym}^{n} h^{1}(B) \cong h^{n}(B)$ for any abelian variety $B$ (here $h^{*}(B)$ refers to the 
Deninger-Murre decomposition of abelian schemes [6]), and so the isomorphism (3) induces an isomorphism

$$
h_{\text {prim }}^{n}(X) \stackrel{\cong}{\rightarrow} \bigoplus_{\chi} h^{n}\left(J_{\chi}\right) \text { in } \mathcal{M}_{\text {rat }} .
$$

The following lemma (which we state separately for possible future reference) now closes the proof of the theorem:

Lemma 2.1. Let $X$ be a smooth projective variety of dimension $n$, and assume that

$$
h(X) \cong \bigoplus_{\chi} h^{n}\left(B_{\chi}\right) \oplus \bigoplus \mathbb{1}(*) \text { in } \mathcal{M}_{\text {rat }}
$$

where $B_{\chi}$ are abelian varieties, and $h^{*}\left(B_{\chi}\right)$ refers to the Deninger-Murre decomposition of the motive of abelian schemes [6]. Then Conjecture 1.1 is true for $X$.

It remains to prove the lemma. (NB: In proving the lemma, we use some results concerning the Chow motive of an abelian variety $B$ and the Beauville decomposition $A_{(*)}^{*}(B)$ of the Chow ring. For a quick survey containing all the results we use, one could look at [24, Section 5].)

Taking Chow groups on both sides of (4), we obtain an isomorphism

$$
A_{\text {hom }}^{n}(X) \stackrel{\cong}{\rightarrow} \bigoplus_{\chi} A^{n}\left(h^{n}\left(B_{\chi}\right)\right) \text {. }
$$

Writing $g_{\chi}:=\operatorname{dim} B_{\chi}$, let us proceed to analyze the various summands in (5):

- In case $n>g_{\chi}$, the summand $A^{n}\left(h^{n}\left(B_{\chi}\right)\right)$ is zero for dimension reasons. The piece $A_{(n)}^{g_{\chi}}\left(B_{\chi}\right)$ of the Beauville decomposition is also zero.

- In case $n=g_{\chi}$, we have that $A^{n}\left(h^{n}\left(B_{\chi}\right)\right)=A_{(n)}^{g_{\chi}}\left(B_{\chi}\right)$, since the Deninger-Murre decomposition $h^{*}(B)$ induces the Beauville decomposition $A_{(*)}^{*}(B)$ in the sense that $A_{(s)}^{j}(B)=A^{j}\left(h^{2 j-s}(B)\right)$ for all integers $j$ and $s$ and any abelian variety $B$.

- Finally, let us assume $n<g_{\chi}$, say $n=g_{\chi}-s$ where $s>0$. In this case, the work of Künnemann [11] provides a "hard Lefschetz" isomorphism of motives

$$
h^{n}\left(B_{\chi}\right) \stackrel{\cong}{\rightarrow} h^{n+2 s}\left(B_{\chi}\right)(s) \text { in } \mathcal{M}_{\text {rat }} .
$$

Taking Chow groups, this induces an isomorphism

$$
A^{n}\left(h^{n}\left(B_{\chi}\right)\right) \stackrel{\cong}{\rightarrow} A^{n+s}\left(h^{n+2 s}\left(B_{\chi}\right)\right)=A_{(n)}^{n+s}\left(B_{\chi}\right)=A_{(n)}^{g_{\chi}}\left(B_{\chi}\right) .
$$

In view of this analysis, we can rewrite (5) in order to have zero-cycles on both sides:

$$
A_{\text {hom }}^{n}(X) \stackrel{\cong}{\rightarrow} \bigoplus_{\chi} A_{(n)}^{g_{\chi}}\left(B_{\chi}\right) \text {. }
$$

We now invoke a result of Vial:

Theorem 2.2 (Vial [27]). Let $B$ be an abelian variety of dimension $g$, and let $\pi_{B}^{j}$ denote the Deninger-Murre projectors [6]. 
(i) Assume $n \in \mathbb{N}$ is even. Then

$$
\left(\wedge^{m} \pi_{B}^{2 g-n}\right)_{*} A_{0}\left(B^{m}\right)=0 \quad \forall m>\left(\begin{array}{l}
g \\
n
\end{array}\right) .
$$

(ii) Assume $n \in \mathbb{N}$ is odd. Then

$$
\left(\operatorname{Sym}^{m} \pi_{B}^{2 g-n}\right)_{*} A_{0}\left(B^{m}\right)=0 \quad \forall m>\left(\begin{array}{l}
g \\
n
\end{array}\right) .
$$

(This is [27, Theorem 4.1]. Vial's result generalizes a result of Voisin [29, Example 4.40], which was the case $n=g$ of Theorem 2.2.)

Armed with Vial's result, we are in position to prove the lemma. Let us treat in detail the case $n$ even (the case $n$ odd is similar and will be left to the zealous reader). Let us write $\pi_{X}^{\text {prim }}$ for the projector such that $\left(X, \pi_{X}^{\text {prim }}, 0\right) \cong \oplus_{\chi} h^{n}\left(B_{\chi}\right)$. Given an integer $m>p_{g}(X)$, using (6) we find

$$
\left(\wedge^{m} \pi_{X}^{\text {prim }}\right)_{*} A_{0}\left(X^{m}\right) \cong \bigoplus_{\sum m_{\chi}=m} \bigotimes_{\chi}\left(\wedge^{m_{\chi}} \pi_{B_{\chi}}^{2 g_{\chi}-n}\right)_{*} A_{0}\left(B_{\chi}^{m_{\chi}}\right)
$$

We note that (4) gives us an equality

$$
p_{g}(X)=\sum_{\chi}\left(\begin{array}{c}
g_{\chi} \\
n
\end{array}\right) .
$$

Since (by assumption) $m>p_{g}(X)$, it follows that in each partition $m=\sum m_{\chi}$ there exists some $\chi$, say $\chi_{0}$, such that

$$
m_{\chi_{0}}>\left(\begin{array}{c}
g_{\chi_{0}} \\
n
\end{array}\right)
$$

Theorem 2.2(i) applied to $B_{\chi_{0}}$ guarantees that in the sum (7) each summand

$$
\bigotimes_{\chi}\left(\wedge^{m_{\chi}} \pi_{B_{\chi}}^{2 g_{\chi}-n}\right)_{*} A_{0}\left(B_{\chi}^{m_{\chi}}\right)
$$

vanishes. It follows that the whole sum (7) vanishes, i.e.

$$
\left(\wedge^{m} \pi_{X}^{\text {prim }}\right)_{*} A_{0}\left(X^{m}\right)=0
$$

Since $\left(\pi_{X}^{\text {prim }}\right)_{*} A_{0}(X)=A_{h o m}^{n}(X)$, this proves Conjecture 1.1 for $X$, in case $n$ is even.

The argument for $n$ odd is similar, the difference being that one considers $\operatorname{Sym}^{m} \pi_{X}^{\text {prim }}$ instead of $\wedge^{m} \pi_{X}^{\text {prim }}$, and one relies on part (ii) of Theorem 2.2 instead of part (i).

Proof. (of Corollary 1.3.) As Voisin had already remarked [28, Corollary 3.5.1], this is implied by the truth of Conjecture 1.1 for $X$ (the implication can be seen using the Bloch-Srinivas argument [4]; this is explained in detail in [18, Corollary 2.7]).

Acknowledgements. Thanks to Kai for enjoyable bike trips in the Alsace countryside. Thanks to the referee for many constructive comments that helped to improve the presentation.

Conflict of interest statement. The author states that there is no conflict of interest. 


\section{REFERENCES}

[1] A. Beauville, Sur l'anneau de Chow d'une variété abélienne, Math. Ann. 273 (1986), 647—651,

[2] G. Bini, R. Laterveer and G. Pacienza, Voisin's conjecture for zero-cycles on Calabi-Yau varieties and their mirrors, Advances in Geometry 20 no. 1 (2020), 91-108,

[3] S. Bloch, Lectures on algebraic cycles, Duke Univ. Press Durham 1980,

[4] S. Bloch and V. Srinivas, Remarks on correspondences and algebraic cycles, American Journal of Mathematics Vol. 105, No 5 (1983), 1235-1253,

[5] D. Burek, Higher-dimensional Calabi-Yau manifolds of Kummer type, arXiv:1810.11084(2018),

[6] Ch. Deninger and J. Murre, Motivic decomposition of abelian schemes and the Fourier transform, J. Reine Angew. Math. 422 (1991), 201-219,

[7] W. Fulton, Intersection theory, Springer-Verlag Ergebnisse der Mathematik, Berlin Heidelberg New York Tokyo 1984,

[8] U. Jannsen, Motivic sheaves and filtrations on Chow groups, in: Motives (U. Jannsen et alii, eds.), Proceedings of Symposia in Pure Mathematics Vol. 55 (1994), Part 1,

[9] U. Jannsen, On finite-dimensional motives and Murre's conjecture, in: Algebraic cycles and motives (J. Nagel and C. Peters, editors), Cambridge University Press, Cambridge 2007,

[10] S. Kimura, Chow groups are finite dimensional, in some sense, Math. Annalen 331 (2005), 173-201,

[11] K. Künnemann, A Lefschetz decomposition for Chow motives of abelian schemes, Invent. Math. 113 no. 1 (1993), 85-102,

[12] R. Laterveer, Some results on a conjecture of Voisin for surfaces of geometric genus one, Boll. Unione Mat. Italiano 9 no. 4 (2016), 435-452,

[13] R. Laterveer, Some desultory remarks concerning algebraic cycles and Calabi-Yau threefolds, Rend. Circ. Mat. Palermo 65 (2) (2016), 333-344,

[14] R. Laterveer, Algebraic cycles on surfaces with $p_{g}=1$ and $q=2$, Comment. Math. Univ. St. Pauli 65 no. 2 (2016), 121-130,

[15] R. Laterveer, Algebraic cycles and Todorov surfaces, Kyoto Journal of Mathematics 58 no. 3 (2018), 493-527,

[16] R. Laterveer, On Voisin's conjecture for zero-cycles on hyperkähler varieties, Journal of the Korean Math. Soc. 54 no. 6 (2017), 1841-1851,

[17] R. Laterveer, Some Calabi-Yau fourfolds verifying Voisin's conjecture, Ricerche di Matematica 67 no. 2 (2018), 401-411,

[18] R. Laterveer, Zero-cycles on self-products of surfaces: some new examples verifying Voisin's conjecture, Rend. Circ. Mat. Palermo, Series 2, 68(2) (2019), 419—431,

[19] R. Laterveer, Zero-cycles on Cancian-Frapporti surfaces, Annali dell'Univ. di Ferrara 65 no. 1 (2019), $127-137$,

[20] R. Laterveer and Ch. Vial, On the Chow ring of Cynk-Hulek Calabi-Yau varieties and Schreieder varieties, Canadian J. of Math. 72 no 2 (2020), 505-536,

[21] J. Murre, On a conjectural filtration on the Chow groups of an algebraic variety, parts I and II, Indag. Math. 4 (1993), 177-201,

[22] J. Murre, J. Nagel and C. Peters, Lectures on the theory of pure motives, Amer. Math. Soc. University Lecture Series 61, Providence 2013,

[23] A.A. Rojtman, The torsion of the group of 0-cycles modulo rational equivalence, Annals of Mathematics 111 (1980), 553-569,

[24] T. Scholl, Classical motives, in: Motives (U. Jannsen et alii, eds.), Proceedings of Symposia in Pure Mathematics Vol. 55 (1994), Part 1,

[25] T. Terasoma, Complete intersections of hypersurfaces-the Fermat case and the quadric case, Japan J. Math. (N.S.) 14 no. 2 (1988), 309-384,

[26] Ch. Vial, Niveau and coniveau filtrations on cohomology groups and Chow groups, Proceedings of the LMS 106(2) (2013), 410—444, 
[27] Ch. Vial, Generic cycles, Lefschetz representations and the generalized Hodge and Bloch conjectures for abelian varieties, Annali della Scuola Normale Superiore di Pisa 21 (2020), 1389-1429,

[28] C. Voisin, Remarks on zero-cycles of self-products of varieties, in: Moduli of vector bundles, Proceedings of the Taniguchi Congress (M. Maruyama, ed.), Marcel Dekker New York Basel Hong Kong 1994,

[29] C. Voisin, Chow Rings, Decomposition of the Diagonal, and the Topology of Families, Princeton University Press, Princeton and Oxford, 2014,

[30] N. Zangani, Algebraic cycles on Todorov surfaces of type (2,12), arXiv:1905.10123(2019).

Institut de Recherche Mathématique Avancée, CNRS - Université de Strasbourg, 7 Rue RENÉ Descartes, 67084 STRAsbourg CEDEX, FRANCE.

E-mail address: robert. laterveer@math.unistra.fr 